\title{
AN EXTENSION OF THE BEURLING-CHEN-HADWIN-SHEN THEOREM FOR NONCOMMUTATIVE HARDY SPACES ASSOCIATED WITH FINITE VON NEUMANN ALGEBRAS
}

\author{
Hainui FAN, Don HADWIN AND WENJING LiU
}

\begin{abstract}
In 2015, Yanni Chen, Don Hadwin and Junhao Shen proved a noncommutative version of Beurling's theorems for a continuous unitarily invariant norm $\alpha$ on a tracial von Neumann algebra $(\mathscr{M}, \tau)$, where $\alpha$ is $\|\cdot\|_{1}$-dominating with respect to $\tau$. In the paper, we first define a class of norms $N_{\Delta}(\mathscr{M}, \tau)$ on $\mathscr{M}$, called determinant, normalized, unitarily invariant continuous norms on $\mathscr{M}$. If $\alpha \in N_{\Delta}(\mathscr{M}, \tau)$, then there exists a faithful normal tracial state $\rho$ on $\mathscr{M}$ such that $\rho(x)=\tau(x g)$ for some positive $g \in L^{1}(\mathscr{Z}, \tau)$ and the determinant of $g$ is positive. For every $\alpha \in N_{\Delta}(\mathscr{M}, \tau)$, we study the noncommutative Hardy spaces $H^{\alpha}(\mathscr{M}, \tau)$, then prove that the Chen-Hadwin-Shen theorem holds for $L^{\alpha}(\mathscr{M}, \tau)$. The key ingredients in the proof of our result include a factorization theorem and a density theorem for $L^{\alpha}(\mathscr{M}, \rho)$.
\end{abstract}

Mathematics subject classification (2010): 46E20, 30H10, 30J99, 47L10.

Keywords and phrases: Gauge norm, von Neumann algebra, Beurling theorem.

\section{REFERENCES}

[1] W. B. ARveson, Analyticity in operator algebras, Amer. J. Math. 89 (1967) 578-642.

[2] T. N. BEKJAN AND Q. XU, Riesz and Szego type factorizations for non-commutative Hardy spaces, J. Operator Theory, 62 (2009) 215-231.

[3] A. Beurling, On two problems concerning linear transformations in Hilbert space, Acta Math. 81 (1949) 239-255.

[4] D. Blecher and L. E. Labuschagne, A Beurling theorem for non-commutative $L^{p}$, J. Operator Theory, 59 (2008) 29-51.

[5] D. Blecher And L. E. Labuschagne, Outers for non commutative $H^{p}$ revisited, arxiv: 1304.0518v1 [math. OA] (2013).

[6] S. Bochner, Generalized conjugate and analytic functions without expansions, Proc. Nat. Acad. Sci. U.S.A. 45 (1959) 855-857.

[7] Y. CHEN, D. HADWIN AND J. SHEN, A non-commutative Beurling's theorem with respect to unitarily invariant norms, arXiv: 1505.03952v1 [math. OA] (2015).

[8] J. DiXMIER, Formes linaires sur un anneau d'oprateurs (French), Bull. Soc. Math. France, 81 (1953) 9-39.

[9] H. FAN, D. HADWIN AND W. LiU, An extension of the Chen-Beurling-Helson-Lowdenslager theorem, arXiv:1611.00357 [math.FA].

[10] U. HAAGERUP AND H. SCHULTZ, Brown measures of unbounded operators affiliated with a finite von Neumann algebra, Math. Scand., 100 (2007), 209-263.

[11] P. Halmos, Shifts on Hilbert spaces, J. Reine Angew. Math. 208 (1961) 102-112.

[12] H. Helson, Lectures on Invariant Subspaces, Academic Press, New York-London, 1964.

[13] H. Helson and D. Lowdenslager, Prediction theory and Fourier series in several variables, Acta Math. 99 (1958) 165-202.

[14] K. Hoffman, Analytic functions and logmodular Banach algebras, Acta Math. 108 (1962) 271-317.

[15] R. V. KADISON AND J. R. RingRose, Fundamentals of the theory of operator algebras, Vol. II: Advanced theory, New York etc., Academic Press 1986. XIV. 
[16] E. Nelson, Notes on non-commutative integration, J. Funct. Anal. 15 (1974) 103-116.

[17] K. S. SAIto, A note on invariant subspaces for finite maximal subdiagonal algebras, Proc. Amer. Math. Soc. 77 (1979) 348-352.

[18] T. P. SRINIVASAN, Simply invariant subspaces, Bull. Amer. Math. Soc. 69 (1963) 706-709.

[19] M. TAKESAKI, Theory of operator algebras I, Springer, 1979. 